\title{
El sistema muscular es el impulsor del desarrollo esquelético
}

\author{
Eckhard Schoenau \\ Hospital Pediátrico, Universidad de Colonia, Colonia, Alemania
}

\author{
Palabras clave \\ Resistencia ósea · Fuerza muscular • Osteoporosis, \\ prevención · Sarcopenia
}

\section{Extracto}

En esta revisión se abordan los conceptos metodológicos de evaluación de la función de la musculatura esquelética y adaptación ósea. Actualmente se considera que la propiedad crítica del hueso no es tanto su peso como su resistencia, y que el control de la resistencia ósea se ejerce principalmente a través del efecto de las cargas mecánicas que inciden sobre el hueso. La contracción muscular representa la carga fisiológica más importante sobre el hueso y, en consecuencia, la estabilidad del hueso debe adaptarse a la fuerza muscular (la unidad funcional músculo-hueso). Las sugerencias y recomendaciones descritas esbozan un concepto nuevo: la masa y la resistencia ósea no estarían relacionadas con la edad. Se dispone cada vez de más pruebas de que la masa y la resistencia óseas estarían relacionadas con la función muscular. Del análisis se infiere que no existiría una entidad denominada 'masa ósea máxima'. Se están realizando actualmente numerosos estudios para evaluar si estos nuevos enfoques incrementan la sensibilidad y la especificidad del pronóstico de fracturas en un individuo. Además, el enfoque de muchos investigadores en la biología ósea se está desplazando desde la masa ósea hasta la geometría ósea o la resistencia ósea y su relación con el impulso del sistema muscular.

Copyright $\odot 2006$ Nestec Ltd., Vevey/S. Karger AG, Basel

\section{Introducción}

Cada generación desafía al sistema de atención sanitaria con nuevas demandas, dado que la prevalencia de los problemas médicos que exigen enfoques preventivos y curativos experimenta cambios constantes. En países occidentales industrializados, la mayoría de los individuos ha sustituido las actividades motoras corrientes previas de la vida cotidiana por la ayuda técnica proporcionada por un mundo mecanizado e informatizado. Dado que el sistema musculoesquelético se está adaptando a los retos biomecánicos y a las condiciones ambientales, la composición corporal del niño y el adolescente medio ha cambiado espectacularmente en las sociedades occidentales $[1,2]$. La resistencia a la insulina, la obesidad, la osteopenia y la sarcopenia son actualmente retos característicos que deben afrontar las autoridades sanitarias que tienen a su cargo la prevención de las enfermedades y la educación sanitaria. Hasta hace unas pocas décadas, la atención sanitaria pediátrica en las sociedades occidentales se centraba en la prevención del raquitismo y en la malnutrición debida a una ingestión calórica deficiente. Actualmente, los problemas urgentes residen en un sistema musculoesquelético atrófico inducido por una actividad motora deficiente y en la malnutrición causada por una ingestión calórica elevada. Además, la mejor asistencia de los niños con enfermedades crónicas ha generado el problema de las enfermedades musculoesqueléticas secundarias. En consecuencia, el conocimiento y la destreza en la evalua-

\section{KARGER}

Fax +4161306 1234

E-Mail karger@karger.ch

www.karger.com (c) 2006 Nestec Ltd., Vevey/S. Karger AG, Basel

$0252-8185 / 06 / 0642-0055 \$ 23.50 / 0$

Accesible online en:

www.karger.com/ans
Prof. Eckhard Schoenau

Children's Hospital, University of Cologne

Kerpener Strasse 62

DE-50924 Cologne (Germany)

Tel. +49221478 4360, Fax +49 221478 3773, E-Mail eckhard.schoenau@uk-koeln.de 
Fig. 1. Modelo funcional de desarrollo óseo basado en la teoría del mecanostato. El componente principal de la regulación es el circuito de retroalimentación entre la deformación ósea (tensión tisular) y la resistencia ósea. Durante el crecimiento, este sistema homeostático es forzado constantemente a adaptarse a los retos externos. Los factores que se muestran en la parte inferior modulan diversos aspectos del sistema regulador central. De Rauch y Schoenau [29], con autorización.

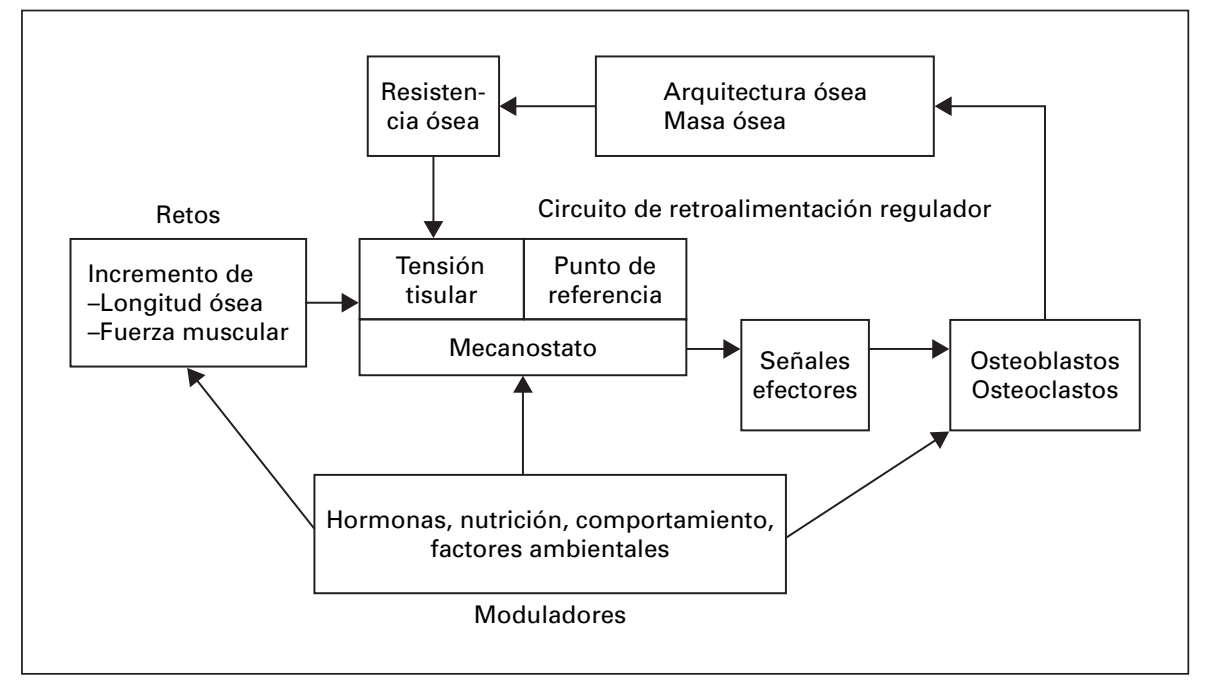

ción de las interacciones musculoesqueléticas se convertirá en algo más que un problema en pediatría. El conocimiento sobre el desarrollo de los sistemas musculoesqueléticos y la evaluación de este proceso llegarán a ser cuestiones importantes para los responsables de la mejoría del nivel de asistencia médica en nuestras sociedades. En esta revisión se discuten las propiedades mecánicas del desarrollo esquelético.

\section{Hipótesis del mecanostato de Harold Frost}

La combinación de los factores que permiten satisfacer las necesidades de los huesos sanos sometidos a carga, de todos los anfibios, aves, mamíferos y reptiles de cualquier tamaño, edad y sexo, recibe el nombre de mecanostato. Combina los mecanismos de modelado y remodelado, sus umbrales, los mecanismos mediadores de la médula ósea, los mecanismos de transmisión de señales que los conectan y, tal vez, otros aspectos. Por razones mecánicas, el sistema de retroalimentación negativo resultante determina cuándo, donde y los huesos sí necesitan más resistencia, o cuándo el hueso no es necesario. Diversos factores extramecánicos, incluyendo hormonas y otros agentes humorales, podrían modular ('ayudar o impedir') los efectos del mecanostato sobre la resistencia ósea. El mecanostato podría ser comparado con la combinación de la dirección, los frenos y el acelerador de un automóvil. Los osteoblastos y los osteoclastos podrían ser análogos a las ruedas del automóvil y al uso mecánico de su conductor (fig. 1) [3-5].
El desarrollo del músculo y el hueso durante el crecimiento es influido por fuerzas asociadas a la gravedad y la actividad física $[4,6,7]$. Las fuerzas musculares son las que crean las fuerzas máximas que actúan sobre el hueso. De este modo, el crecimiento en presencia de descarga actúa tanto en un músculo que carece de capacidad funcional como en un hueso que carece de la forma específica que es única para su función [8]. Esta relación intrínseca entre el músculo y el hueso se describe por la teoría del mecanostato, que sostiene que el incremento de la fuerza muscular máxima durante el crecimiento o en respuesta al incremento de la carga afectará a la masa, al tamaño y a la resistencia del hueso. La descarga (inactividad o inmovilización) dará lugar a la reducción del desarrollo muscular (y la fuerza muscular) y posee un efecto negativo sobre la masa, el tamaño y la resistencia del hueso.

El funcionamiento correcto del mecanostato depende del estado normal de todas sus células (osteocitos, osteoblastos y osteoclastos), el uso mecánico habitual del esqueleto y el entorno endocrino-metabólico [9]. La afinación del mecanostato se consigue mediante puntos de referencia fisiológicos que actúan como umbrales para el inicio o la inhibición del modelado y remodelado óseos. Aunque los puntos de referencia del mecanostato son determinados genéticamente, están regulados por el entorno endocrino. Por ejemplo, se sostiene que la reducción de la concentración de estrógenos incrementa los puntos de referencia para el modelado y el remodelado óseos. El entorno endocrino afecta a la sensibilidad del mecanostato, mediante la cual el hueso adapta su masa, geometría 


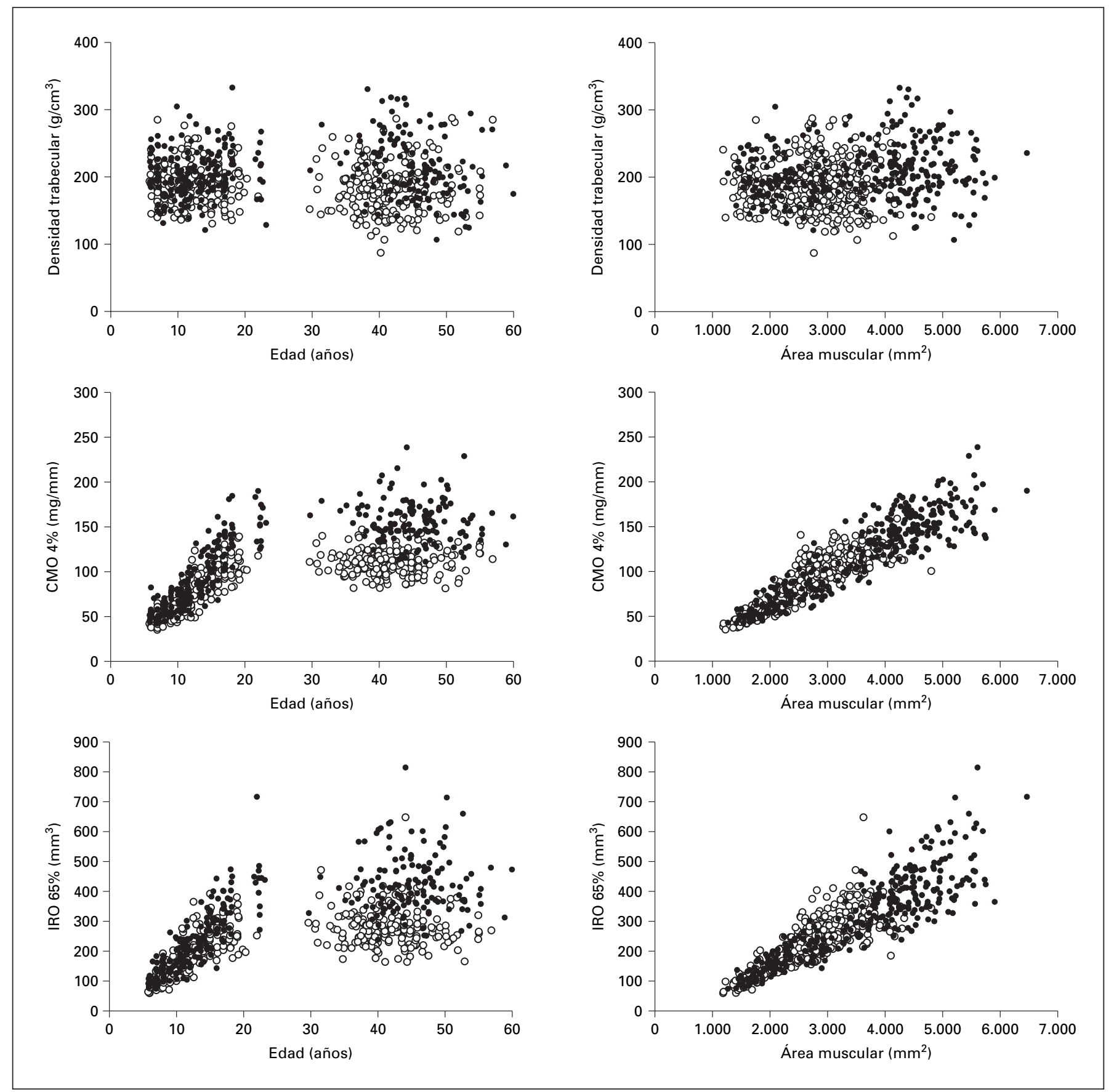

Fig. 2. Densidad trabecular, contenido en mineral óseo (CMO 4\%) e índice de resistencia ósea (IRO 65\%) en relación con la edad y el área muscular. $\bigcirc=$ Hembras; $\mathbf{O}=$ varones. De Schoenau [13], con autorización.

o propiedades estructurales a las deformaciones óseas causadas por la carga [9].

El ejercicio y la nutrición son factores ambientales clave conocidos por afectar al desarrollo muscular y óseo. El ejercicio actúa directamente a través de la acción muscu- lar, e indirectamente a través de la regulación endocrina; durante el crecimiento se considera que el ejercicio influye sobre el modelado óseo y, en consecuencia, sobre la geometría ósea. 


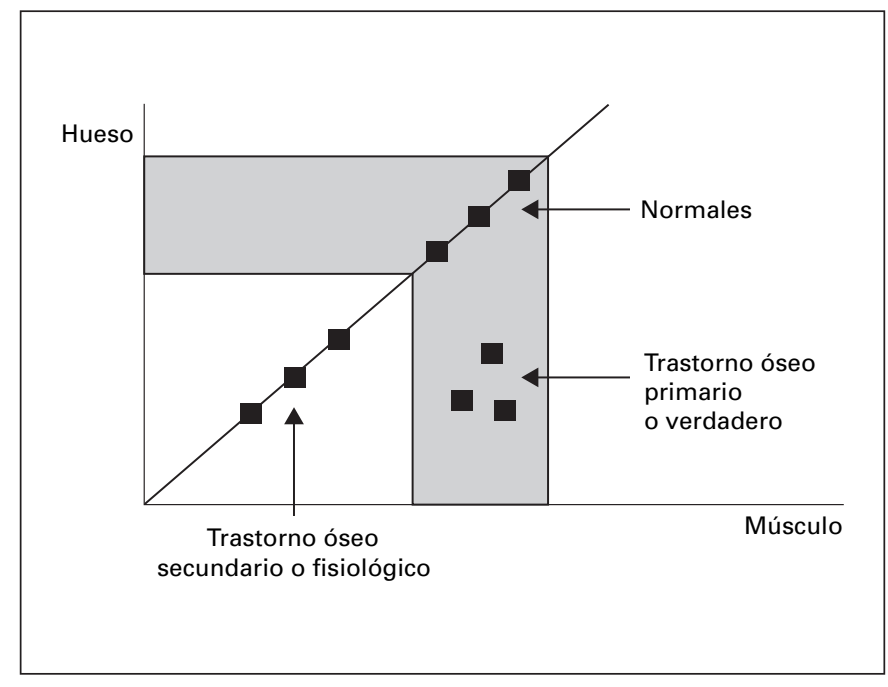

Fig. 3. 'Unidad funcional músculo-hueso'. Osteopatía primaria: estructura/masa óseas no adaptadas al desarrollo muscular. Osteopatía secundaria: desarrollo muscular perturbado, pero esqueleto normalmente adaptado. De Schoenau [13], con autorización.

\section{Desarrollo de la densidad, la masa y la resistencia óseas}

En la figura 2 se presenta la relación entre edad, área muscular y densidad trabecular, masa ósea (contenido en mineral óseo, CMO) y el índice de resistencia ósea (IRO) en una población de referencia sana. Los datos y los resultados antropométricos obtenidos por medio de tomografía computadorizada cuantitativa periférica en estos sujetos han sido descritos previamente [10-12]. La densidad trabecular como índice de densidad tisular no depende de la edad ni del desarrollo muscular. CMO e IRO sólo parecen depender de la edad durante la infancia. En contraste, CMO e IRO muestran una contundente correlación lineal con el desarrollo muscular en la infancia y la edad adulta. Estos datos describen que la densidad ósea es más o menos una 'constante' y CMO e IRO son funciones del desarrollo muscular. Basándose en estas consideraciones, hace algunos años recomendamos relacionar los datos óseos analizados con sucedáneos del desarrollo muscular. En lugar de los datos de referencia relacionados con la edad, el análisis de la llamada 'unidad músculohueso' (fig. 3) debe mejorar el conocimiento de la fisiología y la fisiopatología del desarrollo óseo [13].

\section{Influencia de la pubertad sobre la 'unidad músculo-hueso'}

En 1995, Frost (comunicación personal) formuló la pregunta esencial: ¿Podía el estrógeno, fomentador del crecimiento femenino, añadir más hueso del necesario para actividades físicas con objeto de almacenar calcio extra para gestaciones y lactancias posteriores? Los datos de Zanchetta et al. [14] confirman esta hipótesis. Este grupo de trabajo utilizó la absorciometría de rayos X de energía dual para estimar el CMO corporal total y la masa magra corporal en 778 niños argentinos sanos, de raza blanca. Estos datos fueron analizados nuevamente por Schiessl et al. [15], comprobando que la masa ósea en muchachas durante la pubertad llega a incrementarse más que en muchachos con masas magras corporales similares. En función de las incertidumbres metodológicas y analíticas relacionadas con el uso del CMO total y la masa magra corporal como índices de resistencia ósea y fuerza muscular, y también por la importancia potencial de estos datos, parecía necesaria la realización de estudios adicionales con fines de confirmación. En niños y adolescentes sanos, de edades comprendidas entre 6 y 22 años, así como en adultos que participaron en el Estudio Nutricional y Antropométrico de Diseño Longitudinal de Dortmund, se midieron mediante tomografía computadorizada cuantitativa periférica el área cortical del radio como índice de resistencia ósea y el área muscular representativa de la fuerza muscular $[16,17]$.

Se registró una alta correlación entre el área muscular y el área cortical del radio en todos los niños, adolescentes y adultos. En la figura 4 se ilustran las correlaciones entre el área muscular y el área cortical en varones y hembras. Antes de la pubertad, los chicos y las chicas mostraban la misma relación entre área muscular y área cortical. Sin embargo, transcurrida la pubertad, en las muchachas el área cortical era mayor con respecto al área muscular que en los muchachos. En el estadio puberal 3 podía observarse en las chicas una área cortical relativamente mayor. En la figura 5 se ilustra la relación entre el perímetro perióstico, el perímetro endóstico y el área muscular. La correlación entre área muscular y perímetro perióstico no difería significativamente entre varones y hembras. Por el contrario, se observó una diferencia significativa entre sexos en la relación entre el área muscular y el perímetro endóstico. Estos datos respaldan los conceptos sobre desarrollo óseo durante la infancia y la adolescencia propuestos por Frost y el paradigma de Utah de fisiología esquelética. Las cargas voluntarias más potentes sobre los huesos proceden de los músculos. Para adaptar la resis- 


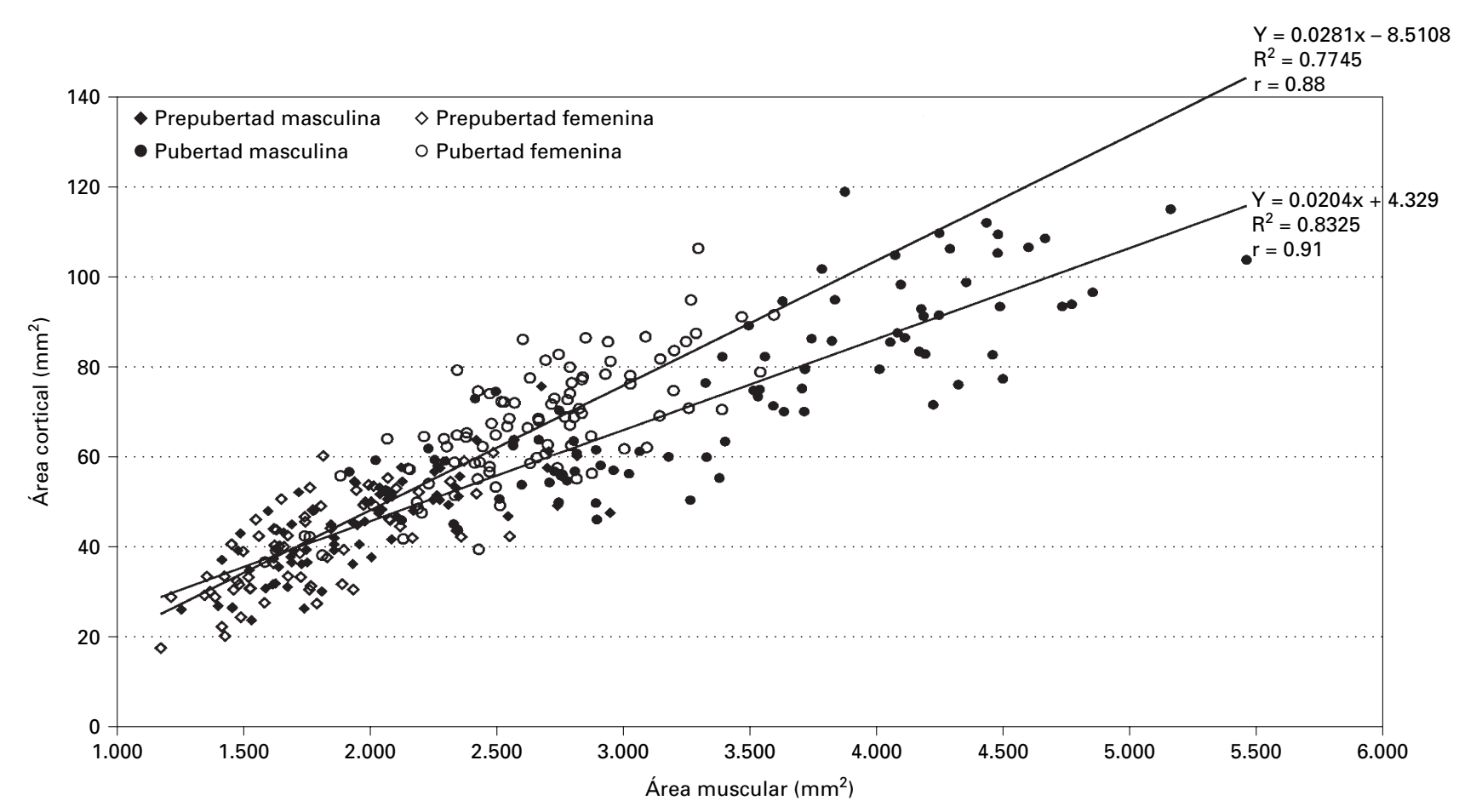

Fig. 4. Área ósea cortical en relación con el área muscular en niños y niñas prepuberales y puberales. Con autorización de Schoenau et al. [16]; copyright 2000, The Endocrine Society.

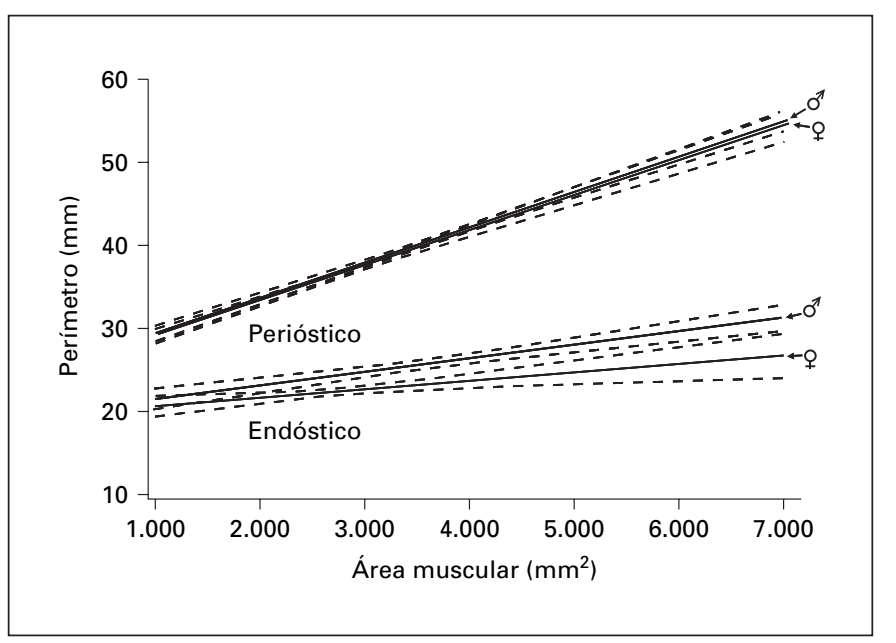

Fig. 5. Rectas de regresión e intervalos de confianza del 95\% de la correlación del área muscular frente a los perímetros perióstico y endóstico. Con autorización de Schoenau et al. [16]; copyright 2000, The Endocrine Society.

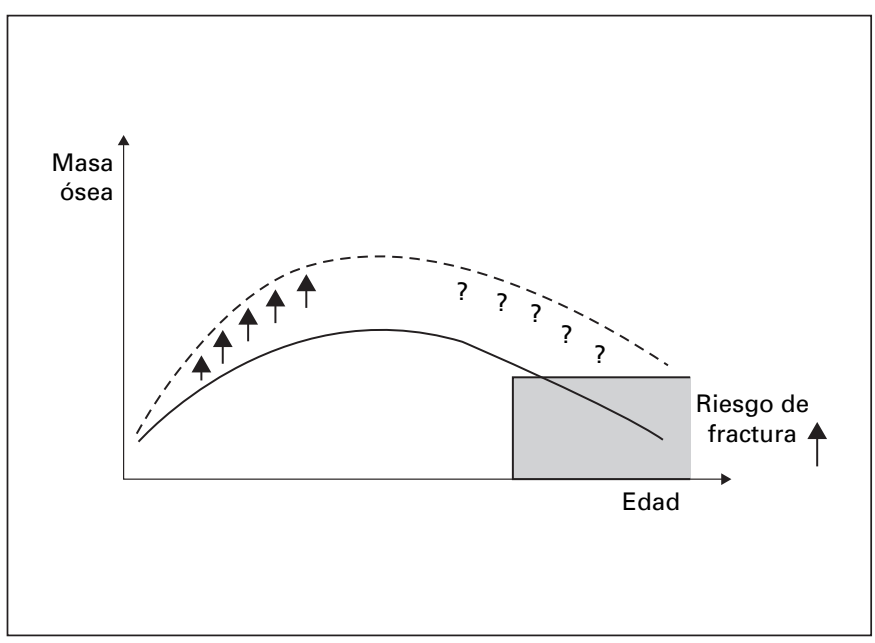

Fig. 6. Concepto de masa ósea máxima: ¿Prevención de fracturas por mejoría de la masa ósea individual durante la infancia y la adolescencia? De Springer Science and Business Media [Pediatr Nephrol 2004;19:825-831], con autorización. 


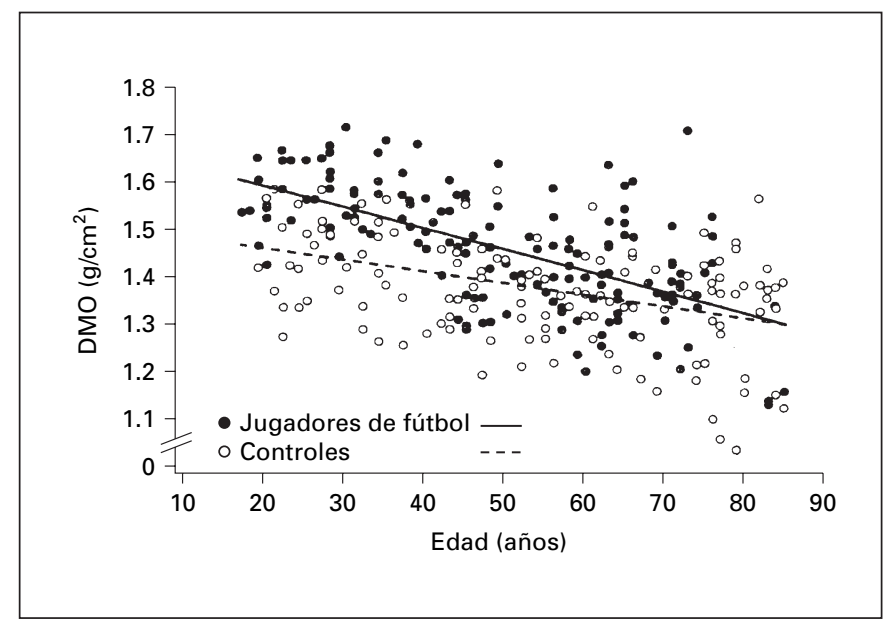

Fig. 7. Densidad del mineral óseo (DMO) en las piernas de jugadores de fútbol en activo y retirados en comparación con sujetos de control. De Johnston et al. [19], con autorización.

tencia y la masa óseas a ellas, intervalos de umbrales de tensión especiales determinan dónde el modelado añade y fortalece el hueso y cuándo el remodelado lo conserva o elimina, del mismo modo que ajustes termostáticos diferentes controlan los sistemas de calentamiento y enfriamiento en una casa. Si los estrógenos afectan la sensibilidad del mecanostato reduciendo el umbral de remodelado durante la pubertad en las chicas, la masa ósea debe comenzar a incrementarse más rápidamente que en los chicos en presencia de fuerzas musculares similares, debido a la reducción de las pérdidas óseas que dependen del remodelado. Los resultados que aquí presentamos complementan los estudios de Zanchetta et al. [14], Schiessl et al. [15] y Ferretti et al. [18], respaldando al mismo tiempo el concepto mencionado.

\section{Masa ósea máxima óptima durante la infancia y la adolescencia: ¿existen efectos a largo plazo?}

Basándose en el concepto, en general aceptado, de adquisición óptima de la masa ósea, en numerosos estudios se ha analizado la influencia de la ingestión de calcio sobre el desarrollo de la masa ósea (fig. 6). Un estudio realizado en gemelos mostraba un efecto positivo, si bien modesto, de una mayor ingestión de calcio sobre la masa ósea en niños [19]. Es interesante destacar que la ingestión de calcio careció de impacto sobre la masa ósea durante la pubertad, a pesar de la extendida creencia de que el suministro de calcio posee una importancia crítica durante el periodo de crecimiento puberal. El seguimiento ulterior de estos gemelos demostró que las diferencias significativas en la densidad del mineral óseo entre el gemelo suplementado con calcio y el gemelo no suplementado con calcio, desaparecían tras la suspensión de los comprimidos de calcio.

En otro estudio se comprobó que 18 meses de aporte complementario controlado de calcio generaban una mayor masa de mineral ósea, si bien estos incrementos desaparecían en el curso de los 18 meses del ensayo [20]. En estudios con marcadores bioquímicos, que reflejan el modelado y el remodelado óseos, se demostró que el consumo de calcio reducía las tasas de remodelado, si bien carecía de influencia sobre el modelado [21].

Esos datos son compatibles con la hipótesis de que el aporte complementario de calcio reduce la actividad de remodelado. Podrían aparecer menores tasas de remodelado a través de la reducción de los niveles de hormona paratiroidea, lo que daría lugar a una menor porosidad cortical. No obstante, este evidente incremento de la masa ósea es completamente reversible tras el incremento de las tasas de remodelado. Cabe destacar por su importancia que el aporte complementario de calcio no parece estimular el modelado, que constituye el proceso principal para incrementar la resistencia ósea (espesor cortical, área cortical) durante la infancia y la adolescencia.

Los efectos a largo plazo de la actividad física pueden también ser reversibles. Un estudio en jugadores de fútbol demostró que si bien el ejercicio en jóvenes confiere un beneficio importante y clínicamente significativo para la densidad máxima del mineral óseo, la consecuencia del cese del ejercicio consiste en pérdidas aceleradas (fig. 7) [22]. Por lo tanto, aquellos que hubieran dejado de jugar durante más de 35 años y que tuvieran 60 o más años no presentarían ningún beneficio residual significativo para la densidad del mineral óseo. La tasa de fracturas no era inferior a la prevista para la edad. Pajamaki et al. [23] informaron de datos similares en un estudio en ratas. Los beneficios óseos inducidos por el ejercicio desaparecen si el ejercicio se suspende completamente y, en consecuencia, un entrenamiento continuado es probablemente necesario para mantener, en la edad adulta, los efectos positivos del ejercicio durante la juventud.

Por otra parte, estudios en animales de experimentación han demostrado que un periodo de adquisición perturbada de la masa ósea en la primera etapa de la vida puede no conllevar una alteración en la masa ósea máxima [24]. El estudio demostró que si bien zonas del esqueleto juvenil no están remodeladas por completo, en reali- 
dad son sustituidas en su totalidad por medio del crecimiento esquelético. A medida que el hueso juvenil se agranda y la cavidad medular se expande, el hueso formado en la primera etapa de la vida, independientemente de la cavidad, es reabsorbido gradualmente y sustituido por hueso nuevo a través del modelado. Este mecanismo 'repara' el desarrollo óseo insuficiente tras el cese de las influencias negativas.

En resumen, los efectos a corto plazo sobre la masa ósea, bien sean positivos o negativos, no parecen producir efecto alguno a largo plazo. El sistema esquelético parece adaptarse más a las exigencias actuales que a las de una fase de desarrollo anterior.

\section{Perspectiva y nuevos conceptos}

Las siguientes sugerencias y recomendaciones esbozan un concepto nuevo, que se basa en las consideraciones anteriores pero cuya certeza no ha sido demostrada en detalle. La masa ósea no estaría relacionada con la edad. Actualmente se dispone cada vez de más pruebas a favor de que la masa ósea estaría relacionada con el tamaño de los huesos [25] o la función muscular [25-31].

Cuando se analiza, el concepto de 'masa ósea máxima' no se sostiene. Actualmente se están realizando diversos estudios para evaluar si estos enfoques incrementan la sensibilidad y la especificidad del pronóstico de fracturas en un individuo.
Además, el enfoque de numerosos investigadores en la biología ósea se está desplazando desde la masa ósea hasta la geometría ósea o la resistencia ósea [32, 33]. La masa ósea es un sucedáneo de la resistencia ósea, si bien pueden utilizarse también técnicas ampliamente disponibles, como la absorciometría de rayos X de energía dual, la radiogrametría y la tomografía computadorizada, para medir variables de geometría ósea como el espesor cortical, el área cortical y el momento de inercia. En estudios futuros se demostrará si el análisis combinado de geometría ósea y masa ósea mejora o elimina el concepto de masa ósea máxima.

En el momento actual, el análisis de la masa ósea es sumamente útil en estudios epidemiológicos sobre factores que pueden ejercer un impacto sobre el desarrollo óseo. No obstante, la masa ósea no puede considerarse un criterio muy sensible y específico del riesgo de fracturas de un individuo. El concepto de masa ósea máxima se basó en la noción de que el desarrollo óptimo durante la infancia y la adolescencia prevendría la aparición de fracturas en las etapas posteriores de la vida adulta. Actualmente es evidente que la presencia de huesos fuertes en los más jóvenes no supone automáticamente una vejez exenta de fracturas. No obstante, cabría la posibilidad de que huesos fuertes seguirían siéndolo si un individuo mantuviese los hábitos de vida sanos que confieren predominantemente fortaleza a los huesos. Queda por ver si esta hipótesis es correcta.

\section{Bibliografía}

$>1$ Strock GA, Cottrell ER, Abang AE, et al: Childhood obesity: a simple equation with complex variables. J Long Term Eff Med Implants 2005;15:15-32.

$\checkmark 2$ Popkin BM: The nutrition transition: an overview of world patterns of change. Nutr Rev 2004;62:S140-S143.

$\checkmark 3$ Frost HM: Bone 'mass' and the 'mechanostat': a proposal. Anat Rec 1987;219:1-9.

$\checkmark 4$ Burr DB: Muscle strength, bone mass, and age-related bone loss. J Bone Miner Res 1997; 12:1547-1551.

5 Schoenau E, Frost HM: The 'muscle-bone unit' in children and adolescents. Calcif Tissue Int 2002;70:405-407.

6 Turner CH: Muscle-bone interactions, revisited. Bone 2000;27:339-340.

7 Frost HM: On our age-related bone loss: insights from a new paradigm. J Bone Miner Res 1997;12:1539-1546.
-8 Frost HM, Schonau E: The 'muscle-bone unit' in children and adolescents: a 2000 overview. J Pediatr Endocrinol Metab 2000;13:571590.

9 Cointry GR, Capozza RF, Negri AL, et al: Biomechanical background for a noninvasive assessment of bone strength and muscle-bone interactions. J Musculoskelet Neuronal Interact 2004;4:1-11.

10 Neu CM, Manz F, Rauch F, et al: Bone densities and bone size at the distal radius in healthy children and adolescents: a study using peripheral quantitative computed tomography. Bone 2001;28:227-232.

11 Neu CM, Rauch F, Manz F, Schoenau E: Modeling of cross-sectional bone size, mass and geometry at the proximal radius: a study of normal bone development using peripheral quantitative computed tomography. Osteoporos Int 2001;12:538-547.
12 Schoenau E, Neu CM, Rauch F, Manz F: The development of bone strength at the proximal radius during childhood and adolescence. J Clin Endocrinol Metab 2001;86:613-618.

13 Schoenau E: From mechanostat theory to development of the 'Functional Muscle-BoneUnit. J Musculoskelet Neuronal Interact 2005; 5:232-238

14 Zanchetta JR, Plotkin H, Alvarez Filgueira ML: Bone mass in children: normative values for the 2-20-year-old population. Bone 1995; 16(suppl):393S-399S.

15 Schiessl H, Frost HM, Jee WS: Estrogen and bone-muscle strength and mass relationships. Bone 1998;22:1-6.

16 Schoenau E, Neu CM, Mokov E, et al: Influence of puberty on muscle area and cortical bone area of the forearm in boys and girls. J Clin Endocrinol Metab 2000;85:1095-1098. 
17 Kersting M, Sichert-Hellert W, Lausen B, et al: Energy intake of 1 to 18 year old German children and adolescents. Z Ernahrungswiss 1998; 37:47-55.

-18 Ferretti JL, Capozza RF, Cointry GR, et al: Gender-related differences in the relationship between densitometric values of whole-body bone mineral content and lean body mass in humans between 2 and 87 years of age. Bone 1998;22:683-690.

19 Johnston CC Jr, Miller JZ, Slemenda CW, et al: Calcium supplementation and increases in bone mineral density in children. N Engl J Med 1992;327:82-87.

20 Lee WT, Leung SS, Leung DM, Cheng JC: A follow-up study on the effects of calcium-supplement withdrawal and puberty on bone acquisition of children. Am J Clin Nutr 1996;64: 71-77.

21 Slemenda CW, Peacock M, Hui S, et al: Reduced rates of skeletal remodeling are associated with increased bone mineral density during the development of peak skeletal mass. J Bone Miner Res 1997;12:676-682.
22 Karlsson MK, Linden C, Karlsson C, et al: Exercise during growth and bone mineral density and fractures in old age. Lancet 2000;355: 469-470.

23 Pajamaki I, Kannus P, Vuohelainen T, et al: The bone gain induced by exercise in puberty is not preserved through a virtually life-long deconditioning: a randomized controlled experimental study in male rats. J Bone Miner Res 2003;18:544-552.

24 Gafni RI, McCarthy EF, Hatcher T, et al: Recovery from osteoporosis through skeletal growth: early bone mass acquisition has little effect on adult bone density. FASEB J 2002;16: 736-738.

25 Kroger H, Vainio P, Nieminen J, Kotaniemi A: Comparison of different models for interpreting bone mineral density measurements using DXA and MRI technology. Bone 1995;17: 157-159.

26 Molgaard C, Thomsen BL, Michaelsen KF: Influence of weight, age and puberty on bone size and bone mineral content in healthy children and adolescents. Acta Paediatr 1998;87: 494-499.
27 Lu PW, Cowell CT, Lloyd-Jones SA, et al: Volumetric bone mineral density in normal subjects, aged 5-27 years. J Clin Endocrinol Metab 1996;81:1586-1590

28 Schoenau E, Neu CM, Beck B, et al: Bone mineral content per muscle cross-sectional area as an index of the functional muscle-bone unit. J Bone Miner Res 2002;17:1095-1101.

29 Rauch F, Schoenau E: The developing bone: slave or master of its cells and molecules? Pediatr Res 2001;50:309-314.

30 Finkelstein JS, Neer RM, Biller BM, et al: Osteopenia in men with a history of delayed puberty. N Engl J Med 1992;326:600-604.

31 Hogler W, Briody J, Woodhead HJ, et al: Importance of lean mass in the interpretation of total body densitometry in children and adolescents. J Pediatr 2003;143:81-88.

32 Seeman E: From density to structure: growing up and growing old on the surfaces of bone. J Bone Miner Res 1997;12:509-521.

33 Seeman E: Periosteal bone formation - a neglected determinant of bone strength. N Engl J Med 2003;349:320-323. 